\title{
Uniform convergence and everywhere convergence of Fourier series. II
}

\section{Masako Izumi and Shin-ichi Izumi}

The first theorem shows that the subspaces of the space of functions with everywhere convergent Fourier series, defined in our previous paper, is a good subspace. The second theorem shows that convergence criterion in the previous paper is the proper generalization of Lebesgue's Convergence Criterion.

\section{Introduction and theorems}

By $e c$ we denote the space of functions, periodic with period $2 \pi$, whose Fourier series converges everywhere.

$L C$ is the space of functions $f$ such that

$$
\int_{0}^{t}(f(x+u)+f(x-u)-2 f(x)) d u=o(t) \text { as } t \rightarrow 0 \text { for all } x
$$

and $M^{p}$ is the space of functions $f$ such that

$$
\int_{-\pi}^{\pi}\left|\Delta_{\pi / n}^{2} f_{1}(u)\right|^{p} d u=o\left(1 / n^{p+1}\right) \text { as } n \rightarrow \infty \text {, }
$$

where $p>1$,

$$
f_{1}(u)=\int_{0}^{u} f(v) d v \text { for all } u \text { in }(-\pi, \pi)
$$

and

$$
\Delta_{\pi / n}^{2} f_{1}(u)=f_{1}(u)-2 f_{1}(u-\pi / n)+f_{1}(u-2 \pi / n) .
$$

Received $16 \mathrm{July} 1973$. 
Further we denote by $N^{P}$ the class of functions $f$ such that

$$
\sum_{m=n}^{\infty}\left(\left|a_{m}\right|^{p}+\left|b_{m}\right|^{p}\right)=o\left(1 / n^{p-1}\right) \text { as } n \rightarrow \infty \text {, }
$$

where $a_{m}$ and $b_{m}$ are the $m$ th cosine and sine coefficients of the Fourier series of $f$.

In the previous paper [2], we have proved the following:

THEOREM A.

(i) ec $\supset L C \cap M^{p}$ for any $p \geq 1$,

(ii) ec $\supset L C \cap N^{p}$ for any $p>1$.

On the other hand we know that

$$
\operatorname{ec} \cap\left(L^{r}-L^{S}\right) \neq \varnothing \quad(1<r<s<\infty)
$$

and

$$
\operatorname{ec} n\left(L^{\infty}-C\right) \neq \varnothing
$$

We shall prove that the subspaces $L C \cap M^{p} \quad(p>1)$ and $L C \cap N^{p}$ $(p>2)$ have the same property as above, that is,

\section{THEOREM 1 .}

(i) $\left(L C \cap M^{p}\right) \cap\left(L^{r}-L^{s}\right) \neq \emptyset \quad(1<p<r<s \leq \infty)$,

(ii) $\left(L C \cap M^{p}\right) \cap\left(L^{\infty}-C\right) \neq \emptyset \quad(p>1)$,

(iii) LC $\cap N^{p} \quad(p \geq 2)$ has the properties (i) and (ii).

This theorem shows that $L C \cap M^{p}$ and $L C \cap N^{p}$ have the first character of "good subspace" (cf. [2]).

On the other hand, there is the well known convergence criterion of Lebesgue:

THEOREM B. If $f$ is an even function satisfying the conditions

$$
f_{1}(t)=\int_{0}^{t} f(u) d u=o(t) \text { as } t \rightarrow 0
$$


and

$$
\int_{2 \pi / n}^{\pi}\left|\Delta_{\pi / n} f(u)\right| \frac{d u}{u}=o(1) \text { as } n \rightarrow \infty \text {, }
$$

where $\Delta_{\pi / n} f(u)=f(u)-f(u-\pi / n)$, then the Fourier series of $f$ converges at the origin.

We have proved the following generalization of Lebesgue's Convergence criterion [2].

THEOREM C. If $f$ is an even function satisfying the conditions (3) and

$$
\int_{3 \pi / n}^{\pi}\left|\Delta_{\pi / n}^{2} f_{1}(u)\right| \frac{d u}{u}=o(1 / n) \text { as } n \rightarrow \infty,
$$

then the Fourier series of $f$ converges at the origin.

Gergen [1] generalized Lebesgue's criterion as follows: the condition (3) is replaced by Cesaro continuity of any positive order $i$, that is,

$$
f_{i}(t)=o\left(t^{i}\right) \text { as } t \rightarrow 0
$$

and the condition (4) by

$$
\int_{(j+1) \pi / n}^{\pi}\left|\Delta_{\pi / n} f(u)\right| \frac{d u}{u}=\bar{o}(1) \text { as } n \rightarrow \infty \text {, }
$$

where $\bar{o}$ is the limit in the Pollard sense (cf. [1]).

Similarly we can generalize Theorem $C$ as follows: the condition (3) can be replaced by $\left(3^{\prime}\right)$ and the condition (5) can be replaced by

$$
\int_{(j+1) \pi / n}^{\pi}\left|\Delta_{\pi / n}^{j} f_{k}(u)\right| \frac{d u}{u}=\bar{o}\left(1 / n^{k}\right) \text { as } n \rightarrow \infty \text {, }
$$

where $\bar{o}$ is the limit in the Pollard sense.

We can easily see that ( $\left.5^{\prime}\right)$ is more general than $\left(4^{\prime}\right)$.

We shall prove that Theorem $C$ is a proper generalization of Lebesgue's and the generalization of Theorem $\mathrm{C}$ is also a proper generalization of Gergen's: 
THEOREM 2. (i) There is a function $f$ satisfying the conditions (3) and (5), but not (4).

(ii) There is a function $f$ satisfying the conditions ( $\left.3^{\prime}\right)$ and (5'), but not (4').

\section{Proof of Theorem 2 (i) and (ii)}

Consider the even and periodic function $f$ defined by

$$
f(u)=u^{-\alpha} \sin l / u \text { on }(0, \pi),
$$

where $0 \leq \alpha<1$.

Then

$$
\begin{aligned}
f_{1}(u) & =\int_{0}^{u} \frac{1}{v^{\alpha}} \sin \frac{1}{v} d v=\int_{1 / u}^{\infty} \frac{\sin v}{v^{2-\alpha}} d v=O\left(u^{2-\alpha}\right) \\
& =o(u) \text { as } u \rightarrow 0
\end{aligned}
$$

and then the condition ( 3 ) is satisfied. On the other hand,

(8) $\Delta_{\pi / n^{2}}^{2} f_{1}(u)=\left(\int_{1 / u}^{1 /(u-\pi / n)}-\int_{1 /(u-\pi / n)}^{1 /(u-2 \pi / n)}\right) \frac{\sin v}{v^{2-\alpha}} d v$

$$
\begin{aligned}
& =\int_{1 / u}^{1 /(u-\pi / n)}\left(\frac{\sin v}{v^{2-\alpha}}-\frac{\sin (v-\pi / n)}{(v-\pi / n)^{2-\alpha}}\right) d v \\
& =-\int_{1 / u}^{1 /(u-\pi / n)} \frac{\sin v}{v^{2-\alpha}(v-\pi / n)^{2-\alpha}}\left\{v^{2-\alpha}-(v-\pi / n)^{2-\alpha}\right\} d v \\
& \qquad \quad+2 \sin \frac{\pi}{2 n} \int_{1 / u}^{1 /(u-\pi / n)} \frac{\cos (v-\pi / 2 n)}{(v-\pi / n)^{2-\alpha}} d v \\
& =O\left(\frac{\pi / n}{u(u-\pi / n)} \cdot \frac{\pi}{n} u^{3-\alpha}\right)+o\left(\frac{\pi}{n} \cdot \frac{\pi / n}{u(u-\pi / n)} \cdot u^{2-\alpha}\right) ;
\end{aligned}
$$

then

$$
\begin{aligned}
\int_{3 \pi / n}^{\pi}\left|\Delta_{\pi / n}^{2} f_{I}(u)\right| \frac{d u}{u} & =o\left(\frac{1}{n^{2}} \int_{3 \pi / n}^{\pi} \frac{d u}{u^{\alpha}}\right)+o\left(\frac{1}{n^{2}} \int_{3 \pi / n}^{\pi} \frac{d u}{u^{1+\alpha}}\right) \\
& =O\left(1 / n^{2-\alpha}\right)=o(1 / n) \text { as } n \rightarrow \infty .
\end{aligned}
$$

Therefore the condition (5) is also satisfied. Now we shall show that the Lebesgue condition (4) is not satisfied. 


$$
\begin{aligned}
\int_{2 \pi / n}^{\pi} & |f(u)-f(u-\pi / n)| \frac{d u}{u}=\int_{2 \pi / n}^{\pi}\left|\frac{1}{u^{\alpha}} \sin \frac{1}{u}-\frac{1}{(u-\pi / n)^{\alpha}} \sin \frac{1}{u-\pi / n}\right| \frac{d u}{u} \\
& \geq \int_{1 / \sqrt{n}}^{\pi}\left|\sin \frac{-\pi / n}{(u-\pi / n)} \cos \frac{2 u-\pi / n}{u(u-\pi / n)}\right| \frac{d u}{u^{\alpha+1}}-\frac{A}{n} \int_{1 / \sqrt{n}}^{\pi}\left|\frac{\sin 1 / u}{u^{2+\alpha}}\right| d u \\
& \geq \frac{A}{n} \int_{1 / \sqrt{n}}^{\pi} \frac{d u}{u^{3+\alpha}}-\frac{A}{n} \int_{1 / \sqrt{n} u^{2+\alpha}}^{\pi} \frac{d u}{\alpha / 2}-\frac{A}{n^{(1-\alpha) / 2}} \geq A \text { for large } n .
\end{aligned}
$$

Thus we have proved Theorem 2 (i).

Theorem 2 (ii) can be proved by the same example.

We shall remark that the sine function of this example can be replaced by the cosine and or the exponential function.

\section{Proof of Theorem 1 (i) and (ii)}

We shall consider the even function $f$ defined by (6). We take

$$
1 / s<\alpha<1 / r<1 \text {, }
$$

then

$$
\int_{0}^{\pi}|f(u)|^{r} d u \leq \int_{0}^{\pi} u^{-\alpha r} d u \leq A
$$

and

$$
\int_{0}^{\pi}|f(u)|^{s} d u \geq A \int_{0}^{\pi} u^{-\alpha s}=\infty
$$

Therefore $f \in L^{r}$ but $f \notin L^{S}$. By (7), $f \in L C$. Now

$$
\int_{-\pi}^{\pi}\left|\Delta_{\pi / n}^{2} f_{I}(u)\right|^{p} d u \leq A \int_{0}^{3 \pi / n}+A \int_{3 \pi / n}^{\pi}=P+Q
$$

where

$$
P \leq A \int_{0}^{3 \pi / n}\left|f_{1}(u)\right|^{p} d u=A \int_{0}^{3 \pi / n} u^{(2-\alpha) p} d u \leq A / n^{(2-\alpha) p+1}=o\left(1 / n^{p+1}\right)
$$

by (7) and 


$$
\begin{aligned}
Q & \leq A \int_{3 \pi / n}^{\pi}\left|\Delta_{\pi / n}^{2} f_{1}(u)\right|^{p} d u \leq A \frac{1}{n^{2 p}} \int_{3 \pi / n}^{\pi} \frac{d u}{u^{\alpha p}} \leq A / n^{(2-\alpha) p+1} \\
& =o\left(1 / n^{p+1}\right)
\end{aligned}
$$

by (8). Therefore $f \in M^{p}$. Thus we have proved Theorem 1 (i).

For the proof of Theorem I (ii), we use the same function (6) with $\alpha=0$. Then this function does not belong to the space $C$ and belongs to the spaces $L C \cap M^{p}$ and $L^{\infty}$.

\section{References}

[1] J.J. Gergen, "Convergence and summability criteria for Fourier series", Quart. J. Math. Oxford 1 (1930), 252-275.

[2] Masako Izumi and Shin-ichi Izumi, "Uniform convergence and everywhere convergence of Fourier series. I", Bull. Austral. Math. Soc. 9 (1973), 321-335.

Department of Mathematics,

Institute of Advanced Studies,

Australian National University,

Canberra, ACT. 